\title{
Can Cold Brew Coffee be Convenient? A Pilot Study for Caffeine Content in Cold Brew Coffee Concentrate Using High Performance Liquid Chromatography
}

\author{
Sarah Lane, Josh Palmer, Dr. Brian R. Christie, Dr. Juergen Ehlting, Cuong H. Le* \\ University of Victoria \\ slane@uvic.ca
}

\begin{abstract}
Cold brew coffee is a brewing method that is increasing in prevalence. While it has been anecdotally suggested that this method may provide a more aromatic and flavourful coffee product, there is little research published that looks at the concentration of caffeine or other coffee substituents in cold brew coffee. The potential alteration in chemical composition in cold brew provides a few interesting avenues for research. Can caffeine in cold brew be quantified by conventional methods? If so, how does the caffeine profile of cold brews relate to hot brew methods? Here we report the caffeine content and variability in small batch cold brew coffee and show that HPLC/UV-Vis, a standard method for quantitation of caffeine in other extraction methods, is useful for detection of caffeine in cold brew coffee. The mean concentration of caffeine in an average $355 \mathrm{~mL}$ serving was found to be $207.22 \pm 39.17 \mathrm{mg}$ over five distinct batches of cold brew coffee concentrate. Cold brew preparation methods produce similar quantities of caffeine as hot brew preparation, yet may have increased storage capabilities including improved retention of flavonoids and other secondary metabolites. Therefore, cold brew may provide utility in clinical trials examining caffeine and the effect of other components of coffee as it is commonly consumed.
\end{abstract}

Keywords: Cold Brew; Coffee; Caffeine; HPLC; Extraction;

Orof affeine is North America's most popular psychoactive drug and is consumed at a dietary level by $90 \%$ of the USA's population (Frary, Johnson, \& Wang, 2005; Heckman, Weil, Mejia, \& Gonzalez, 2010). Caffeine is a naturally occurring alkaloid, and its popularity in part stems from its actions as a generalized stimulant through non-selective inhibition of adenosine receptors in the brain (Fisone, Borgkvist, \& Usiello, 2004; Nehlig, Daval, \& Debry, 1992). This inhibition, alongside systemic vasodilation and improved calcium intake, results in a boost to physical, psychomotor, and cognitive abilities and improvements to subjective factors, such as mood and alertness (Einother \& Giesbrecht, 2013; Ferré, 2008). Since the primary source of caffeine intake for adult populations is coffee, it is important to consider caffeination effects in a dietarily relevant vehicle (Wanyika, Gatebe, Gitu, Ngumba, \& Maritim, 2010). However, coffee as a beverage category shows a large variability in caffeine quantity between preparation methods, and not all are ideally suited for research purposes.

In general, caffeine content is dependent on the brew water temperature, length of contact time, extraction pressure, grind size of bean, coffee bean roast method, the Coffea species, and cultivar the beans originated from (Barone \& Roberts, 1995; Wanyika et al., 2010). Ultimately, these factors

${ }^{*}$ This work was supported by an NSERC Discovery grant to Juergen Ehlting. The authors are also very appreciative of Sam Jones of $2 \%$ Jazz Coffee for the generous donation of cold brew concentrate used in this study. 
are chosen to reflect both cultural and individual preferences of flavour, aroma, and caffeine content (Gloess et al., 2013). A unifying factor across most brewing processes is the use of hot water to improve the solubility of many coffee constituents (Farah, 2012). However, coffee can be brewed using cold water if the grounds are given adequate time to steep. This extraction method, coined "cold brew," has experienced a recent surge in popularity, breaking into the inventory of major coffee distributors within the last two years ("Nariño 70 Cold Brew," 2017). Cold brew extraction utilizes lukewarm to refrigerated water and steeps the coffee grinds for upwards of 12 hours. The low temperature better retains volatile organic components, and therefore the coffee maintains its flavour in long term storage or in open environments. These compounds are difficult to study in hot extraction methods, as the high temperature of water necessary for brewing increases the volatility of many of the flavourful secondary metabolites and causes them to be lost or altered (Albanese, Di Matteo, Poiana, \& Spagnamusso, 2009; Salamanca, Fiol, González, Saez, \& Villaescusa, 2017). This change to the brewing process will alter both the profiles of caffeine and other secondary psychoactive metabolites, as well as flavour and stability of the product.

Despite an increase in the prevalence of cold brew coffee, to our knowledge at the time of writing, few peer-reviewed studies have been published examining the relative amount of caffeine, or secondary psychoactive components, in cold brews. Although the cold brew market has increased by $580 \%$ between 2011 and 2016, much of it is in the ready-to-drink category, which is often bottled with additional ingredients that are not ideal for research purposes (Barry, 2016; Sisel, 2016). Smaller producers that make it in-house are therefore potentially more appropriate, but small batches may lead to more variability. This study examines if caffeine content in cold brew can be adequately quantified by HPLC/UV-Vis, the standard method of detecting caffeine in hot extractions, and how caffeine content from cold brew concentrate prepared by an independent small-batch coffee company relates to general recommended dosages per serving published from other extraction methods (Cano-Marquina, Tarín, \& Cano, 2013).

\section{Materials and Methods}

\section{Reagents}

Methanol (MeOH, HPLC grade) and formic acid (FA, reagent grade) were purchased from Caledon Laboratories Ltd (Georgetown, ON, CA), and acetonitrile (ACN, HPLC grade) was purchased from Fisher Scientific (Markham, ON, CA). Double distilled water was purified by a Super-Q water filtration system (model ZDPP02254, Millipore, Bedford, MA, USA) before use. Caffeine standard for the purpose of instrument calibration was obtained from ESA Biosciences (model 70-6565, analytical grade, Chelmsford, MA, USA).

\section{Apparatus}

Analysis was performed using high performance liquid chromatography (HPLC), and based on standard methods such as those reported by Naegele (2013). Method was developed for caffeine determination of cold brew concentrate on a Dionex UltiMate 3000 (model HPG-3400A, Thermo Fischer Scientific, Waltham, MA, USA) equipped with a solvent rack with inline degasser (model SRD-3400, Thermo Fischer Scientific), a binary high pressure gradient pump (model HPG-3400, Thermo Fischer Scientific), and an autosampler (model ACC-3000T, Thermo Fischer Scientific) with a $20 \mu \mathrm{L}$ sample loop. A UV-Vis photodiode array (model PDA-3000, Thermo Fischer Scientific) with a flow cell pathlength of $10 \mathrm{~mm}$ was used to detect analytes. The column was a Kinetex Reverse Phase C18 (2.6 m; $100 \AA \AA, 150$ x 4.6 mm, model 00F-4462-E0, Phenomenex, Torrance, CA, USA). 
The binary mobile phase was A: $0.4 \%$ FA in $\mathrm{ddH}_{2} \mathrm{O}(\mathrm{v} / \mathrm{v})$ and B: $0.4 \% \mathrm{FA}$ in ACN (v/v). Elution was a gradient of $0-10 \mathrm{~min}, 80: 20(\mathrm{~A}: \mathrm{B}), 10-20 \mathrm{~min} 50: 50$, at a flow rate of $0.5 \mathrm{~mL} / \mathrm{min}$, with a column temperature of $30^{\circ} \mathrm{C}$. Full loop sample injection was controlled at $10{ }^{\circ} \mathrm{C}$ with an injection volume of $20 \mu \mathrm{L}$, and the detection wavelength was $272 \mathrm{~nm}$.

\section{Sample Preparation}

Five individual batches of cold brew coffee were prepared independently by $2 \%$ Jazz Coffee (Victoria, BC, CA) according to their proprietary standard method on randomly selected days. A $500 \mathrm{~mL}$ sample of each batch was collected and stored at $4^{\circ} \mathrm{C}$ until preparation of sample for analysis. One $\mathrm{mL}$ of each sample was loaded onto a Strata ${ }^{\mathrm{TM}}$-X Polymeric Reverse-Phase column (model 8B-S100-FBL, Phenomenex), eluted in $1 \mathrm{~mL} \mathrm{MeOH}$, and diluted 1:20 in $\mathrm{ddH}_{2} \mathrm{O}$, before running the sample on the HPLC. Samples were run in triplicate.

\section{Determination of Linearity, Recovery, and Calibration}

A standard curve was prepared by dilution of an analytical grade caffeine standard (model 70-6565, ESA Biosciences) with an original concentration of $0.250 \mathrm{mg} / \mathrm{mL}$ to $0.200 \mathrm{mg} / \mathrm{mL}, 0.150$ $\mathrm{mg} / \mathrm{mL}, 0.100 \mathrm{mg} / \mathrm{mL}, 0.050 \mathrm{mg} / \mathrm{mL}$ and $0.025 \mathrm{mg} / \mathrm{mL}$ in $\mathrm{ddH}_{2} \mathrm{O}$, which was run in triplicate. Matrix effects of other components in coffee on caffeine recovery were assessed by method of standard addition. $0.075 \mathrm{~mL}$ of coffee sample was spiked with an analytical grade caffeine standard and diluted to $0.150 \mathrm{~mL}$ with $\mathrm{ddH}_{2} \mathrm{O}$ to achieve a total spike concentration of $0 \mathrm{mg} / \mathrm{mL}, 0.020 \mathrm{mg} / \mathrm{mL}$, $0.040 \mathrm{mg} / \mathrm{mL}, 0.060 \mathrm{mg} / \mathrm{mL}, 0.080 \mathrm{mg} / \mathrm{mL}$ and $0.100 \mathrm{mg} / \mathrm{mL}$. Limit of detection was determined using a serially diluted low range standard curve of $0.040 \mathrm{mg} / \mathrm{mL}, 0.020 \mathrm{mg} / \mathrm{mL}, 0.010 \mathrm{mg} / \mathrm{mL}$, $0.005 \mathrm{mg} / \mathrm{mL}, 0.0025 \mathrm{mg} / \mathrm{mL}$, and $0.00125 \mathrm{mg} / \mathrm{mL}$. Standard error, LOQ, and LOD were calculated using Microsoft Excel 2016 as outlined by Shrivastava and Gupta (2011).

\section{Results and Discussion}

The analysis of caffeine in cold brew coffee reported here was based on HPLC, the separation of chemical compounds that, when paired with an ultraviolet/visible light detector, allows quantification of these compounds by measuring light absorption as they exit the column and are eluted. In this study, the wavelength used was $272 \mathrm{~nm}$, close to the absorption maximum of caffeine. For absolute quantification, this was compared to an analytical grade caffeine standard analyzed under the same conditions. Retention time for the caffeine standard was $4.5 \mathrm{~min}$, and it had absorbance maxima at $275.7 \mathrm{~nm}$ and $231.0 \mathrm{~nm}$ (Figure 1). 


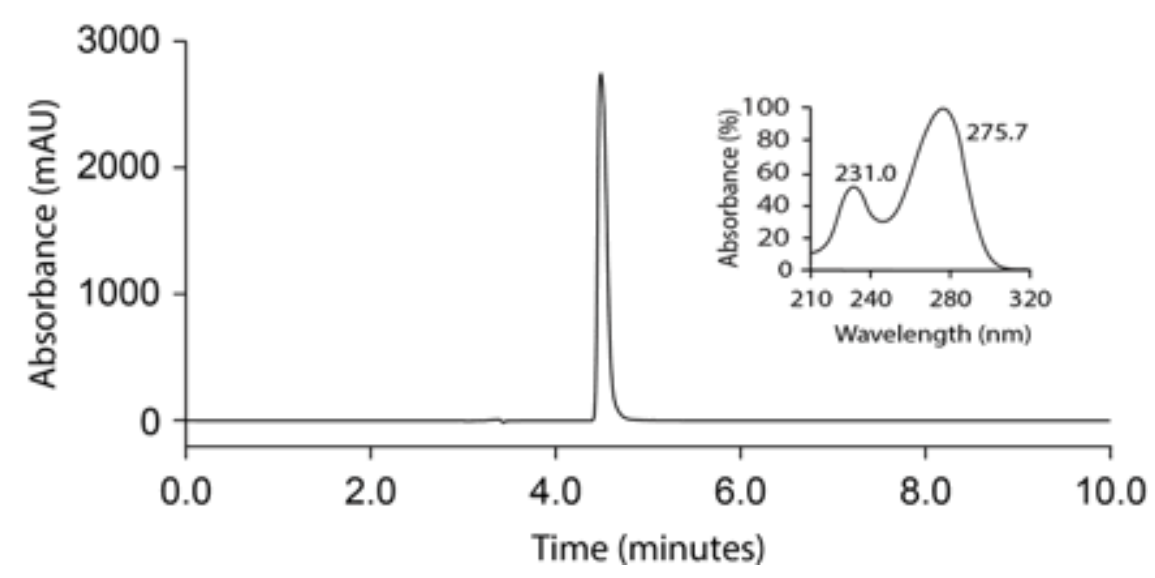

Figure 1. HPLC trace of $0.100 \mathrm{mg} / \mathrm{mL}$ caffeine standard measured at an absorbance wavelength of $272 \mathrm{~nm}$; inset of UV trace for caffeine peak obtained from 3D chromatogram. Caffeine standard has a retention time of $4.5 \mathrm{~min}$; UV trace shows $\lambda^{\max }$ at $231.0 \mathrm{~nm}$ and 275.7 $\mathrm{nm}$.

Employing various caffeine concentrations, the linear range for the detection method was observed to be between $0.025 \mathrm{mg} / \mathrm{mL}$ and $0.150 \mathrm{mg} / \mathrm{mL}$, with an $\mathrm{R}^{2}$ value of 0.9904 (Figure 2). This indicates very high linearity within this range. The limit of detection (LOD) was $0.000527 \mathrm{mg} / \mathrm{mL}$, whose
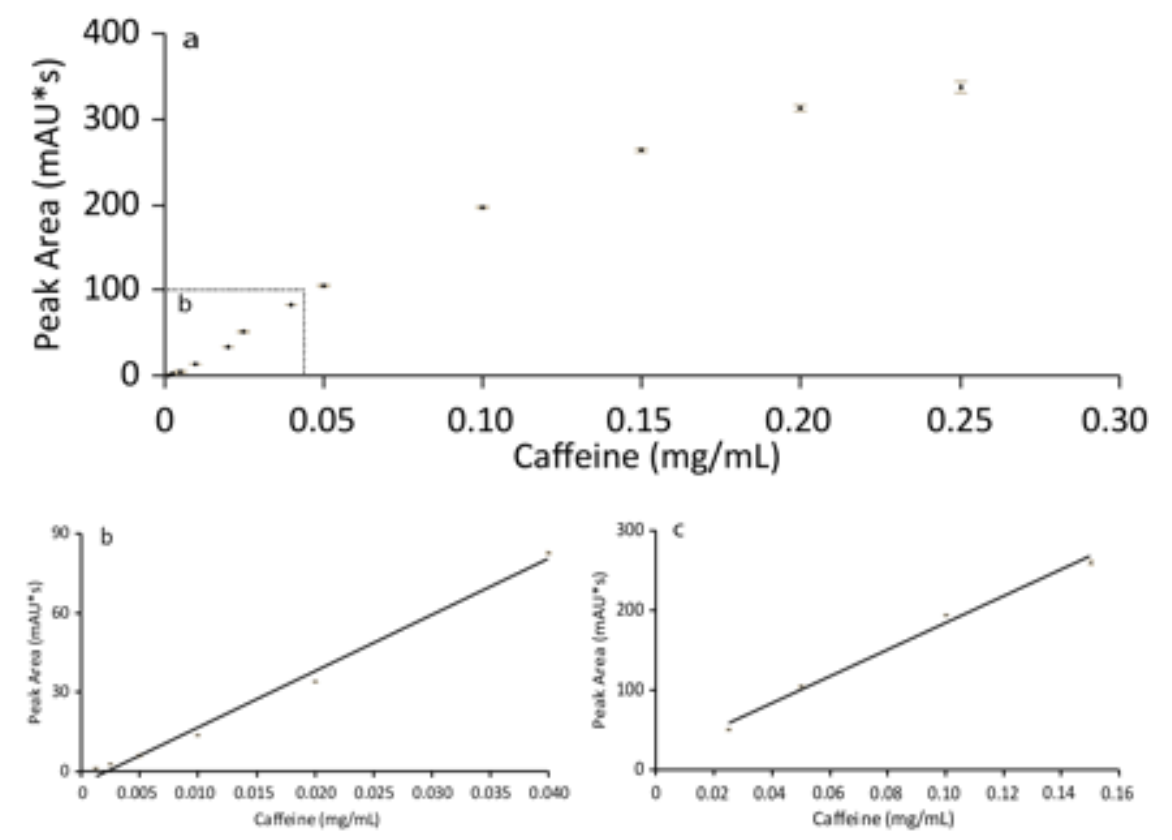

Figure 2. a. Complete range of caffeine concentrations analysed during development of method used to quantify caffeine in cold brew coffee samples. b. Lower range of caffeine concentrations used in determination of $L O D$ and $L O Q$ scores. $L O D: 0.000527 \mathrm{mg} / \mathrm{mL} ; L O Q: 0.00160$ $\mathrm{mg} / \mathrm{mL}$. c. Linear range of the calibration curve used for quantification of caffeine in cold brew coffee samples. Linear equation is $y=1698 x+16.889, R^{2}=0.9904$ 
value is determined from parameters of the regression line of the calibration curve (Shrivastava \& Gupta, 2011). The limit of quantitation (LOQ) was $0.00160 \mathrm{mg} / \mathrm{mL}$ by a similar method and is an indication of the lowest measurement that can be obtained accurately. This was at the lowest part of the linear region, further suggesting that quantifications within the range of the standard curve are highly reliable. To assess technical variability, replicate injections of pure caffeine at a concentration of $0.100 \mathrm{mg} / \mathrm{mL}$ were performed. The coefficient of variation was $1.20 \%$, with a mean observed concentration of $0.1069 \pm 0.0008 \mathrm{mg} / \mathrm{mL}$. To assess effects of other compounds in coffee extracts, pure caffeine was added to a sample of coffee at a final spike concentration ranging from 0 $\mathrm{mg} / \mathrm{mL}$ to $0.100 \mathrm{mg} / \mathrm{mL}$ (Figure 3 ). This process establishes the amount of caffeine that can be

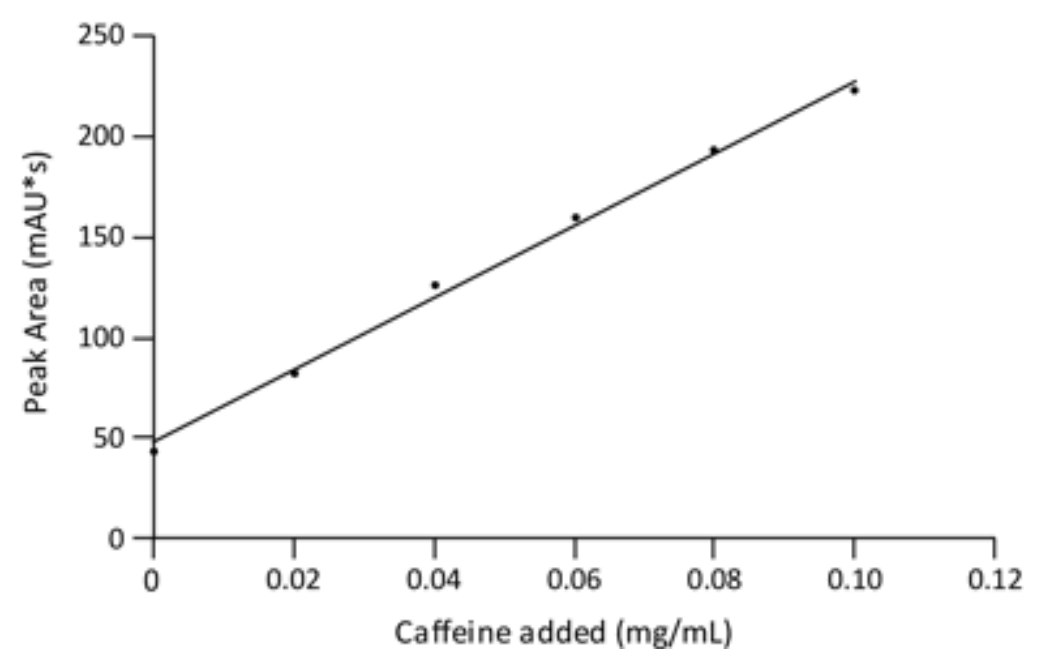

Figure 3. Method of standard addition curve for recovery of caffeine under the matrix influences of cold brew coffee concentrate. Linear equation is $y=1797.9 x+48.409 ; R^{2}=0.9963$.

recovered from coffee by considering other potential molecular interactions that might affect how caffeine is detected. The relationship between all concentrations tested showed a linear increase in caffeine detected $\left(\mathrm{R}^{2}=0.9963\right)$, which suggests that the matrix effect of coffee's other components on the recovery of caffeine is consistent over this range. The recovery of the caffeine spike under matrix effects was $97.4 \%$, sample spike concentration of $0.100 \mathrm{mg} / \mathrm{mL}$ caffeine. This suggests losses owing to experimental parameters were negligible.

A total of five independent cold-brew coffee batches were analyzed. All samples showed a distinct HPLC peak eluting at the same time as the caffeine standard (4.5 min) (Figure 4). The absorption spectrum of this peak closely resembles that of the standard (compare insets in Figures 1 and 4), thereby identifying this peak as caffeine. Additional peaks were detected (Figure 4), which eluted at different times and showed distinct absorption profiles when compared to the caffeine standard. These signals thus refer to other compounds present in the coffee samples, which were not further investigated here. The average caffeine content per batch was $1.1678 \pm 0.2207 \mathrm{mg} / \mathrm{mL}$. To assess the amount of caffeine per serving, caffeine was normalized to the average volume of cold brew concentrate used by $2 \%$ Jazz Coffee (1:1 concentrate to water ratio) in the making of a $355 \mathrm{~mL}$ (12 fl. oz.) beverage. The mean caffeine content per serving was $207.22 \pm 39.17 \mathrm{mg}$ (Table 1). This is within the range of values reported by other companies manufacturing cold brew coffee drinks when normalized to the same serving size. One analysis of Swiss Water ${ }^{\circledR}$ brand coffee reported their caffeine content of cold brew concentrate in a 1:2.268 (concentrate to water) dilution as equivalent to $238 \mathrm{mg}$ (Strumpf, 2015). Starbucks ${ }^{\circledR}$ reported their cold brew beverage contained 


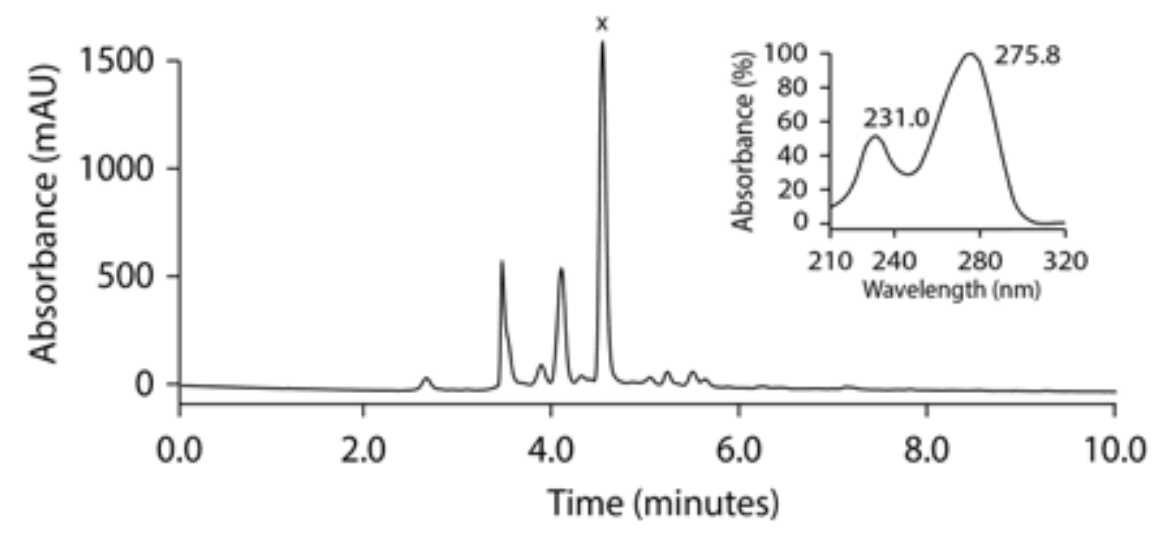

Figure 4. HPLC trace of a representative coffee sample measured at an absorbance wavelength of 272 $\mathrm{nm}$; inset of $U V /$ Vis trace for caffeine peak $(x)$ obtained from $3 D$ chromatogram. Peak $x$ has a retention time of $4.5 \mathrm{~min}$; $U V$ trace shows $\lambda^{\max }$ at $231.0 \mathrm{~nm}$ and $275.8 \mathrm{~nm}$, which is consistent with parameters observed for the caffeine standard (see Fig. 1)

$150 \mathrm{mg}$ per serving ("Nariño 70 Cold Brew," 2017). The value of caffeine reported for cold brew here is also consistent with reported values for caffeine brewed using hot extraction methods (de Mejia \& Ramirez-Mares, 2014; Gloess et al., 2013). When reported values were adjusted to a 355 $\mathrm{mL}$ (12 fl. oz.) serving size, the caffeine content for regular drip coffee was 142.5-495 $\mathrm{mg}$, and for a single espresso (29.6 mL, 1 fl. oz.) caffeine content was 75-225 mg (de Mejia \& Ramirez-Mares, 2014). In another study, espresso had an equivalent caffeine content of $\sim 63 \mathrm{mg}$, but that varied by machine (Gloess et al., 2013).

Cold brew coffee in this study showed some variability between batches, with a standard deviation of $39.17 \mathrm{mg}$ over five samples and a range of $99.36 \mathrm{mg}$, normalized to serving size (Table 1). The

\section{Table 1}

Caffeine content in individual coffee concentrate batches prepared using standard cold brew method from 2\% Jazz Coffee.

\begin{tabular}{c|cc} 
Batch & Caffeine Content $(\mathrm{mg} / \mathrm{mL})$ & Caffeine Content per Serving $(\mathrm{mg} / 355 \mathrm{~mL})$ \\
\hline 1 & $1.3408 \pm 0.0143$ & $237.74 \pm 2.54$ \\
3 & $1.1069 \pm 0.0028$ & $196.41 \pm 0.50$ \\
4 & $0.8619 \pm 0.0027$ & $152.94 \pm 0.47$ \\
5 & $1.1087 \pm 0.0042$ & $196.73 \pm 0.75$ \\
\hline Average & $1.1678 \pm 0.2207$ & $207.22 \pm 39.17$
\end{tabular}

Note: Batches were independently brewed on randomly selected days and stored at $4^{\circ} \mathrm{C}$ until tested. Caffeine content per serving is normalized to $355 \mathrm{~mL}$ (12 fl. oz.) to represent the standard volume of concentrate used by $2 \%$ Jazz Coffee in the preparation of cold brewed coffee for the consumer.

average percentage variance per serving is $14.6 \pm 9.5 \%$. This variance agrees with studies that suggest variation in aroma, flavour, and other components of coffee is natural and based on grind, roast, and other factors that are difficult to account for (Barone \& Roberts, 1995; Wanyika et al., 
The Arbutus Review $\bullet 2017 \bullet$ Vol. 8, No. 1 • http://dx.doi.org/10.18357/tar81201716816

2010). However, the range of caffeine content for other coffee preparations, $150 \mathrm{mg}$ for a single espresso and $352.5 \mathrm{mg}$ for brewed coffee, is significant as reported by de Mejia and Ramirez-Mares (2014). This value is much greater than the range reported here for cold brew, $99.36 \mathrm{mg}$, which is suggestive of a much narrower variance in cold brew than in hot extractions. If the batch variance in cold brew production could be minimized further, then cold brew concentrate may have a significant advantage over hot preparation methods as a research tool.

\section{Conclusion}

Here we show that caffeine content in small batch cold brew can be quantified by HPLC/UV-Vis and when normalized to a serving proportion is similar in caffeine content to various hot coffee brews. The variability in small batches reflects the variable nature of both the roasting and brewing process, but remains within the range of caffeine deemed acceptable for public consumption (Cano-Marquina et al., 2013). Because cold brew concentrate has the potential for improved shelf life, better retention of secondary metabolites and volatiles, and can be brewed in volume, it may be useful in a research setting as a reliable source of caffeine in a commonly ingested form. Future studies should look at characterising the components of cold brew coffee that are not retained in hot extractions and their effect on the consumer. Other studies might examine the kinetics of caffeine release, optimal brew time, and other variables that contribute to the batch variability observed in this study, which would improve its potential for use in clinical caffeine trials. 
The Arbutus Review $\bullet 2017 \bullet$ Vol. 8, No. 1 • http://dx.doi.org/10.18357/tar81201716816

\section{References}

Albanese, D., Di Matteo, M., Poiana, M., \& Spagnamusso, S. (2009). Espresso coffee (EC) by POD: Study of thermal profile during extraction process and influence of water temperature on chemical-physical and sensorial properties. Food Research International, 42(5-6), 727-732. http://doi.org/10.1016/j.foodres.2009.02.027

Barone, J. J., \& Roberts, H. R. (1996). Caffeine consumption. Food and Chemical Toxicology, 34(1), $119-129$.

Barry, D. (2016 December 28). Competition heats up in the cold brew category. Food Business News. Retrieved from http://www.foodbusinessnews.net/articles/news_home/Business_News/2016 /12/Competition_heats_up_in_the_co.aspx?ID=\%7B24ABF61D-320F-46FB-B713-B5CFB $2967789 \% 7 \mathrm{D}$

Cano-Marquina, A., Tarín, J., \& Cano, A. (2013). The impact of coffee on health. Maturitas,75(1), 7-21. doi:10.1016/j.maturitas.2013.02.002

de Mejia, E. G., \& Ramirez-Mares, M. V. (2014). Impact of caffeine and coffee on our health. Trends in Endocrinology 83 Metabolism, $25(10)$, 489-492.

Einöther, S. J., \& Giesbrecht, T. (2012). Caffeine as an attention enhancer: Reviewing existing assumptions. Psychopharmacology, 225(2), 251-274. doi:10.1007/s00213-012-2917-4

Farah, A. (2012). Coffee constituents. In Y. Chu (Eds.), Coffee: Emerging health effects and disease prevention, (pp. 22-58). Retrieved from https://books.google.ca/books?id=y0qA89vCr3MC\& printsec $=$ frontcover $\& d q=$ coffee + emerging + health + effects + and + disease + prevention $\&$ hl $=$ en $\& z$ sa $=$ X\&redir_esc=y\#v=onepage\&q=coffee $\% 3 \mathrm{~A} \% 20$ emerging $\% 20$ health $\% 20$ effects $\% 20$ and $\% 20$ disease\%20prevention\&f=false

Ferré, S. (2008). An update on the mechanisms of the psychostimulant effects of caffeine. Journal of Neurochemistry, 105(4), 1067-1079.

Fisone, G., Borgkvist, A., \& Usiello, A. (2004). Caffeine as a psychomotor stimulant: Mechanism of action. Cellular and Molecular Life Sciences, 61(7), 857-872.

Frary, C. D., Johnson, R. K., \& Wang, M. Q. (2005). Food sources and intakes of caffeine in the diets of persons in the United States. Journal of the American Dietetic Association, 105(1), $110-113$.

Gloess, A. N., Schönbächler, B., Klopprogge, B., Lucio, D., Chatelain, K., Bongartz, A., ...\& Yeretzian, C. (2013). Comparison of nine common coffee extraction methods: Instrumental and sensory analysis. European Food Research and Technology, 236(4), 607-627.

Heckman, M. A., Weil, J., Mejia, D., \& Gonzalez, E. (2010). Caffeine (1, 3, 7-trimethylxanthine) in foods: A comprehensive review on consumption, functionality, safety, and regulatory matters. Journal of Food Science, 75(3), R77-R87.

Naegele, E. (2013). Determination of Caffeine in Coffee Products According to DIN 20487. Application Note Agilent, 1-6. Retrieved from https://www.agilent.com/cs/library/applications/59 91-2851EN.pdf

Nariño 70 Cold Brew. (2017, April 29). Retrieved fromhttps://www.starbucks.com/menu/drinks/ brewed-coffee/cold-brew-coffee\#size=11044804

Nehlig, A., Daval, J. L., \& Debry, G. (1992). Caffeine and the central nervous system: Mechanisms of action, biochemical, metabolic and psychostimulant effects. Brain Research Reviews, 17(2), $139-170$.

Salamanca, C. A., Fiol, N., González, C., Saez, M., \& Villaescusa, I. (2017). Extraction of espresso coffee by using gradient of temperature. Effect on physicochemical and sensorial characteristics of espresso. Food Chemistry, 214, 622-630. http://doi.org/10.1016/j.foodchem.2016.07.120 
Shrivastava, A., \& Gupta, V. (2011). Methods for the determination of limit of detection and limit of quantitation of the analytical methods. Chronicles of Young Scientists, 2(1), 21-25. doi:10.4103/2229-5186.79345

Sisel, E. (2016 July 29). The strength of cold brew. Mintel. Retrieved from http://www.mintel.com/ $\mathrm{blog} /$ drink-market-news/the-strength-of-cold-brew

Strumpf, M. (2015 July 14). Keep your cool with cold brew. Specialty Coffee Chronicle. Retrieved from http://scaa.org//2015/07/14/keep-your-cool-with-cold-brew/

Wanyika, H. N., Gatebe, E. G., Gitu, L. M., Ngumba, E. K., \& Maritim, C. W. (2010). Determination of caffeine content of tea and instant coffee brands found in the Kenyan market. African Journal of Food Science, 4(6), 353-358. 